Pure and Applied Mathematics Quarterly

Volume 4, Number 1

(Special Issue: In honor of

Gregory Margulis, Part 2 of 2$)$

$127-145,2008$

\title{
Proximal Linear Maps
}

\author{
Herbert Abels \\ Dedicated to G. Margulis on the occasion of his 60th birthday
}

\section{INTRODUCTION}

Let $V$ be a finite dimensional vector space over $\mathbb{R}$. A linear endomorphism $g$ of $V$ is called proximal if it has a unique eigenvalue of maximal absolute value and this eigenvalue has algebraic (and hence geometric) multiplicity one. Equivalently, $g$ is proximal iff there is a $g$-invariant line $L^{+}$with $g v=\lambda v$ for every $v \in L^{+}$and a $g$-invariant hyperplane $H^{-}$such that all the eigenvalues of $g$ on $H^{-}$have absolute value smaller than $|\lambda|$. Then $V=L^{+} \oplus H^{-}$. If $g$ is proximal, the eigenvalue $\lambda$ is called the dominant eigenvalue of $g$.

For a proximal element $g \in G L(V)$ the dynamics of the semigroup $g^{n}, n \in \mathbb{N}$, are very transparent. The properties are most conveniently formulated in terms of the map $\mathbb{P} g$ induced by $g$ on the projective space $\mathbb{P}(V)$ corresponding to $V$. Namely, if $g$ is proximal then on $\mathbb{P}(V) \backslash \mathbb{P}\left(H^{-}\right)$the sequence of maps $(\mathbb{P} g)^{n}$, $n \in \mathbb{N}$, converges to the constant map, which sends all of $\mathbb{P}(V) \backslash \mathbb{P}\left(H^{-}\right)$to the point $\mathbb{P}\left(L^{+}\right)$. The convergence is uniform on compact subsets of $\mathbb{P}(V) \backslash \mathbb{P}\left(H^{-}\right)$.

Proximal linear maps have been used in many contexts. Let us mention a few. The linear map of the Perron theorem turns out to be proximal, see section 3, cf. $[\mathrm{BP}],[\mathrm{G}]$ and $[\mathrm{HJ}]$ for applications and generalizations. Tits used proximal maps to obtain a ping-pong situation for a Schottky construction of free subgroups of linear groups, see $[\mathrm{T}]$ and theorem 3.4. Similar constructions were used in [MS], in our work on the Auslander conjecture [A, AMS1-5] and in the work of Breuillard and Gelander [BG]. See also the survey paper [S] of G. Soifer in this volume. Furstenberg used proximal linear maps to study proximal actions in his theory of boundaries [Fu], which in turn played an important role in Margulis' proof of the arithmeticity and superrigidity theorems, see $[\mathrm{M}]$. Other applications

Received May 25, 2006. 
of the concept of proximality to boundary theory and random walks are in the work of Guivarc'h and coauthors. Interesting new applications of the concept of proximality are in the work of Benoist and his students. Proximal linear maps were also used in Gol'dsheid-Margulis' paper to analyze products of random matrices [GM]. The proof of theorem 5.1 is taken from that paper. An essential tool in this proof are quasiprojective maps, introduced by Furstenberg in the paper cited above. We review this concept briefly in chapter 4 .

In fact, this expository paper has as its main purpose to popularize the notion of proximality and to draw attention to the theorem of Gol'dsheid and Margulis. The proof of their very basic algebraic theorem, theorem 5.1 in our paper, is in their paper $[\mathrm{GM}]$ somewhat hiding behind the notion of contractive sequence and some probabilistic applications. The relation between contractive sequences and proximality is described below, see the end of chapter $4,4.10 \mathrm{ff}$.

The notion of proximality, although very useful, seems very special. But by using exterior products questions about maps with multiple eigenvalues of maximal modulus and more generally about Lyapunov filtrations can be reduced to questions about proximality, see the discussion in [AMS1, chapter 5], cf. 5.5.

The word proximal denotes the opposite of the word distal. The notion of a distal action was defined already in Hilbert's Grundlagen der Geometrie. This concept as well as the opposite notion of proximal actions were studied by Furstenberg. From there the notion of a proximal map is derived.

Let me take this opportunity to thank G. Margulis. It has been a great experience to work together with him and I owe him many insights. It was encouraging to see him believe in ever so weird conjectures that finally turned out right.

Acknowledgment: I thank the SFB 701 in Bielefeld, the Clay Mathematical Institute and the Institute of Advanced Study in Princeton for support and Yale University and the IAS for their hospitality during the preparation of this paper.

\section{Elementary properties of PROXimal maps}

2.1. If $n$ is a positive integer, then $g$ is proximal iff $g^{n}$ is proximal. In which case $\lambda\left(g^{n}\right)=(\lambda(g))^{n}, L^{+}\left(g^{n}\right)=L^{+}(g)$ and $H^{-}\left(g^{n}\right)=H^{-}(g)$.

2.2. Let $h \in \operatorname{End}(V)$ have $\operatorname{rank} 1$. Then $h$ is proximal iff $\operatorname{Im}(h) \not \subset \operatorname{Ker}(h)$. In this case $L^{+}=\operatorname{Im}(h)$ and $H^{-}=\operatorname{Ker}(h)$.

2.3. The set of proximal elements in $\operatorname{End}(V)$ is an open subset with respect to the Euclidean topology, but not with respect to the Zariski topology. For an example, $g \in S L_{2}(\mathbb{R})$ is proximal iff $g$ is hyperbolic iff the trace of $g$ has modulus $>2$. And the set of hyperbolic elements of $S L_{2}(\mathbb{R})$ is Euclidean open and Zariski dense in $S L_{2}(\mathbb{R})$. 
2.4. Here is a partial converse of 2.2 and 2.3. If $g$ is proximal with dominant eigenvalue $\lambda$, then $g_{n}=\frac{g^{n}}{\lambda^{n}}$ converges to a linear map as in 2.2. Namely, $g_{n} \mid L^{+}=$ $\operatorname{id}_{L^{+}}$and $g_{n} \mid H^{-}$converges to 0 .

\section{The theorems of Perron and Tits}

An important question is how to find proximal linear maps. We present here three theorems answering this question, due to Tits, Perron and Gol'dsheidMargulis, resp.

A theorem with many applications is Perron's theorem, see [BP], [G] and [HJ]. We present here a geometric version of it.

A convex cone is called pointed if it does not contain an affine line.

Theorem 3.1. Let $C$ be a pointed closed convex cone in $V$ and let $A$ be a linear endomorphism of $V$ such that $A^{m}(C \backslash\{0\}) \subset \stackrel{\circ}{C}$ for some positive integer $m$, where $\stackrel{\circ}{C}$ is the interior of $C$. Then $A$ is proximal, the dominant eigenvalue $\lambda$ is positive and has an eigenvector $x$ in $\stackrel{\circ}{C}$, and the $A$-invariant hyperplane $H$ which is complementary to $\mathbb{R} x$ intersects $C$ only in 0 .

Perron's original theorem is the case that $C$ is the cone of vectors in $\mathbb{R}^{n}$ with non-negative coordinates. So $A$ satisfies the hypotheses of the theorem iff all entries of the corresponding matrix are positive. Thus:

Theorem 3.2 (Perron). Let $A$ be a real $n \times n$-matrix all of whose entries $a_{i j}$ are positive : $a_{i j}>0$ for $i, j=1, \ldots, n$. Then the linear map induced by $A$ is proximal, its dominant eigenvalue $\lambda$ is positive and has a corresponding eigenvector with all entries positive. Furthermore, there is an A-invariant hyperplane $H$ with the following properties: The only vector of $H$ with non-negative entries is the zero vector and the eigenvalues of $A$ on $H$ all have modulus less than $\lambda$.

Again, it suffices that some positive power $A^{m}$ of $A$ fulfills these hypotheses, by 2.1. The more general theorem of Frobenius admits also matrices with nonnegative entries but needs an irreducibility condition. We will not go into the details here. As a warning see the example in 4.10.

Our proof of theorem 3.1 is an application of the Banach fixed point theorem. I thank G. Noskov for showing me this proof $[\mathrm{N}]$. The metric we use here is the Hilbert metric. For the relevant facts about the Hilbert metric see the appendix. Given a pointed closed convex cone $C$ in $V$, then there is an affine hyperplane $H$ in $V$ not containing 0 such that $H \cap C$ is compact and $C$ consists of the nonnegative multiples of vectors of $H \cap C$. Suppose $H$ is given by the linear form $\ell$ on $V: H=\{v \in V ; \ell(v)=1\}$. We may suppose that $m=1$ in the hypothesis 
of 3.1, by 2.1. Put $\widetilde{C}=C \cap H$ and $\widetilde{A}: \widetilde{C} \rightarrow \widetilde{C}, \widetilde{A}(v)=\frac{A(v)}{\ell(A(v))}$. Note that for $v \neq 0, v \in C$ we have $\ell(A(v))>0$, since $A(v) \in \stackrel{\circ}{C} \subset C \backslash\{0\}$ and $\ell$ is positive on $C \backslash\{0\}$. Then $\widetilde{A}(\widetilde{C})$ is compact and contained in the interior of $\widetilde{C}$, hence $\widetilde{A}$ is a contraction of $\widetilde{C}$ with respect to the Hilbert metric, by 6 b) of the appendix. Thus, by the Banach fixed point theorem

(1) $\widetilde{A}$ has a unique fixed point $x$ in $\widetilde{C}$, which must be an element of $\widetilde{A}(\widetilde{C}) \subset \stackrel{\circ}{C}$, and

(2) $\widetilde{A}^{n} \widetilde{y}$ converges to $x$ for every $\widetilde{y} \in \widetilde{C}$.

These two properties imply our claims. First of all, $x$ is an eigenvector for $A$ for the positive eigenvalue $\lambda=\ell(x)$. Every $A$-invariant vector subspace of $V$ which contains a non-zero vector of $C$ must contain $x$, by (2). It thus remains to show that $\lambda$ is the only eigenvalue of modulus $\lambda$, has geometric and algebraic multiplicity 1 and has maximal modulus among all eigenvalues. All of this is also implied by (2), as follows. Suppose there is an eigenvalue of modulus $>\lambda$. Let $f(t)=\prod_{i=1}^{n}\left(t-\lambda_{i}\right)$ be the characteristic polynomial of $A, \Omega=\left\{\lambda_{i} ;\left|\lambda_{i}\right|>\right.$ $\lambda\}, f_{1}(t)=\prod_{\lambda_{i} \in \Omega}\left(t-\lambda_{i}\right)$ and $W=$ ker $f_{1}(A)$. Look at the projective space $\mathbb{P}(\mathbb{R} x \oplus W)$, the map $\mathbb{P}(A)$ induced by $A$ and the point $\mathbb{P}(y)$ corresponding to a line $\mathbb{R} y \neq \mathbb{R} x$. Then the cluster points of $\mathbb{P}(A)^{n} \mathbb{P}(y)$ are all in $\mathbb{P}(W) \not \supset$ $\mathbb{P}(x)$, which contradicts the fact that $\mathbb{P}(A)^{n} \mathbb{P}(y)$ converges to $\mathbb{P}(x)$ for $y \in \widetilde{C}$ near $x$. If the eigenspace of $\lambda$ has dimension at least two or if there is another eigenvalue, real or complex, of modulus $\lambda$, then there is a one or two-dimensional $A$-invariant subspace $W$ not containing $x$ on which $\lambda^{-1} A$ acts by rotation and hence $\lambda^{-n} A^{n}(x+w), w \in W$, remains in a compact subset not intersecting $\mathbb{R} x$, if $w \neq 0$, again contradicting (2). Finally, if $\lambda$ is an eigenvalue with algebraic multiplicity at least two but only one-dimensional eigenspace $\mathbb{R} x$, then there is a two-dimensional $A$-invariant subspace $W$ containing $\mathbb{R} x$ with the following dynamics of $A$. Let $\|\cdot\|$ be a norm on $W$ and let $S$ be the norm-1-sphere $S=\{w \in W ;\|w\|=1\}$, a circle. Define $A^{\prime}: S \rightarrow S, A^{\prime}(x)=\frac{A(x)}{\|A(x)\|}$. Then $A^{\prime}$ has two fixed points on $S$, namely $\pm \frac{x}{\|x\|}$ and on one of the remaining two half circles $A^{\prime} n w$ converges to $\frac{x}{\|x\|}$ for $n \rightarrow \infty$ and on the other one $A^{\prime} n w$ converges to $\frac{-x}{\|x\|}$, which again contradicts $(2)$.

3.3. The following theorem is due to Tits $[\mathrm{T}]$. It holds for arbitrary local fields, not only for $\mathbb{R}$. To state it we need the concept of an admissible metric. Thus, let $k$ be a locally compact field endowed with an absolute value $\omega$. The topology we consider here on a finite dimensional vector space $V$ over $k$ and on the projective space $P=\mathbb{P}(V)=(V \backslash\{0\}) / k^{*}$ is the one induced from the topology of $k$. Thus the topology of $V$ is the product topology for some linear isomorphism $V \simeq k^{n}$ and the topology of $P$ is the quotient topology of the topology of $V \backslash\{0\}$. 
If $x=\left(x_{1}, \ldots, x_{n}\right)$ is an affine coordinate system in some affine space $A$ over $k$, in particular a linear coordinate system in a vector space, we denote by $d_{x}$ : $A \times A \rightarrow \mathbb{R}$ the function defined by

$$
d_{x}(p, q)=\sup _{1 \leq i \leq m}\left(\omega\left(x_{i}(p)-x_{i}(q)\right)\right) .
$$

If $\xi=\left(\xi_{0}, \ldots, \xi_{n}\right)$ is a (linear) coordinate system in the vector space $V$ over $k$, the equation $\xi_{0}=0$ defines a hyperplane $H$ of the projective space $\mathbb{P}(V)$ and $x_{i}=$ $\xi_{0}^{-1} \xi_{i}$ can be viewed as a function on $P \backslash H$. Any such system $x=\left(x_{1}, \ldots, x_{n}\right)$ will be called an affine coordinate system in $P$. The set $D_{x}:=P \backslash H$ will be called its "domain of definition".

A metric $d: P \times P \rightarrow \mathbb{R}_{+}$is called admissible if it induces the topology of $P$ and if for every affine coordinate system $x$ in $P$ and every compact subset $K$ of $D_{x}$ there exist $m, M \in \mathbb{R}_{+}^{*}$ such that

$$
\left.m \cdot d_{x}\right|_{K \times K} \leq\left. d\right|_{K \times K} \leq\left. M \cdot d_{x}\right|_{K \times K} \cdot
$$

Let $X$ be a set, $Y$ a subset, $d: X \times X \rightarrow \mathbb{R}_{+}$a metric and $f: Y \rightarrow X$ a mapping. Then we denote by $\|f\|_{d}$ the "norm of $f$ with respect to $d$ ", that is the number

$$
\sup _{\substack{p, q \in Y \\ p \neq q}} \frac{d(f(p), f(q))}{d(p, q)},
$$

where we set $\|f\|_{d}=0$ if $\operatorname{card} Y \leq 1$.

Let us fix an admissible metric $d$ on $P$.

Theorem 3.4 ([T $3.8(\mathrm{ii})])$. Let $g \in P G L(P)$ and $K \subset P$ be a compact set. Let $\stackrel{\circ}{K}$ be the interior of $K$ in $P$. Suppose that for some $m \in \mathbb{N}$ we have $g^{m} K \subset \stackrel{\circ}{K}$ and $\left\|\left.g^{m}\right|_{K}\right\|_{d}<1$. Then every linear map representing $g$ is proximal. Furthermore, let $L^{+}$be the eigenline of its dominant eigenvalue and let $H^{-}$be the complementary invariant hyperplane, and let $\mathbb{P} L^{+}$and $\mathbb{P} H^{-}$be the corresponding point and hyperplane in $P$. Then $\mathbb{P} L^{+} \in \stackrel{\circ}{K}$ and $\mathbb{P} H^{-} \cap K=\emptyset$.

\section{Quasiprojective MAPS}

A basic tool for the Gol'dsheid-Margulis theorem and their proof are quasiprojective maps. The definition of quasiprojective maps is due to H. Furstenberg. His original definition is contained in 4.8. The definition given here is more general and more categorical.

For a real vector space $V$ we denote by $\mathbb{P} V$ the projective space of onedimensional vector subspaces of $V$. Let $V$ and $W$ be finite dimensional real vector spaces. Let $q: V \rightarrow W$ be a linear map. Then $q$ does not induce a projective map $\mathbb{P} V \rightarrow \mathbb{P} W$, in general, but does so on $\mathbb{P} V \backslash \mathbb{P K e r} q$. We thus define $\mathbb{P}(q): \mathbb{P} V \backslash \mathbb{P}$ Ker $q) \rightarrow \mathbb{P} W$ by $\mathbb{P}(q)(\mathbb{R} x)=\mathbb{R} q(x)$ for every $x \in V \backslash$ Ker $q$. 
Definition 4.1. A map $Q: \mathbb{P} V \rightarrow \mathbb{P} W$ is called quasiprojective if for every non-zero vector subspace $U$ of $V$ there is a non-zero linear map $q_{U}$ such that $Q \mid \mathbb{P} U \backslash \mathbb{P} K e r q_{U}=\mathbb{P} q_{U}$. We then say that $q_{U}$ represents $Q$ on $U$.

4.2. For $Q$ to be quasiprojective it suffices to have linear maps $q_{U}$ representing $Q$ for a sequence of subspaces $U=V_{i}, i=0, \ldots$ constructed as follows: $V_{0}:=$ $V, q_{0}:=q_{V}, V_{1}:=\operatorname{Ker} q_{0}, q_{1}:=q_{V_{1}}: V_{1} \rightarrow W, V_{2}:=\operatorname{Ker} q_{1}, q_{2}:=q_{V_{2}}:$ $V_{2} \rightarrow W$, etc. $V_{i+1}:=\operatorname{Ker} q_{i}, q_{i+1}:=q_{V_{i+1}}$ up to some $q_{r}: V_{r} \rightarrow W$ with $V_{r+1}:=\operatorname{Ker} q_{r}=\{0\}$. If we have a map $Q: \mathbb{P} V \rightarrow \mathbb{P} W$ and such a sequence of subspaces $V_{i}$ and linear maps $q_{i}$, such that $Q \mid \mathbb{P} V_{i+1}=\mathbb{P} q_{i}$ for $i=0, \ldots, r$ then $Q$ is quasiprojective, since for every non-zero vector subspace $U$ of $V$ there is a unique index $i$ such that $U \subset V_{i}$ but $U \not \subset V_{i+1}$. If we put $q_{U}:=q_{i} \mid U$, the system of $q_{U}$ 's has the required properties.

4.3. If $Q$ is a quasiprojective map $Q: \mathbb{P} V \rightarrow \mathbb{P} W$ and $R$ is a quasiprojective map $R: \mathbb{P} W \rightarrow \mathbb{P} U$ then $R \circ Q \mid \mathbb{P} V \rightarrow \mathbb{P} U$ is a quasiprojective map, because for a non-zero vector subspace $V^{\prime}$ of $V$ we have $R \circ Q \mid \mathbb{P} V^{\prime} \backslash \mathbb{P} V^{\prime \prime}=\mathbb{P}\left(r_{W^{\prime}} \circ q_{V^{\prime}}\right)$, where $W^{\prime}=q_{V^{\prime}}\left(V^{\prime}\right)$ and $V^{\prime \prime}=\operatorname{Ker}\left(r_{W^{\prime}} \circ q_{V^{\prime}}\right)<V^{\prime}$ and $q_{V^{\prime}}$ and $r_{W^{\prime}}$ are the linear maps representing $Q$ and $R$ on $V^{\prime}$ and $W^{\prime}$, resp. Thus, projective spaces together with quasiprojective maps form a category.

4.4. For a quasiprojective map $Q: \mathbb{P} V \rightarrow \mathbb{P} W$ let us define $M_{0}(Q) \subset \mathbb{P} W$ as the $Q$-image of the set of points where $Q$ is continuous and $M_{1}(Q) \subset \mathbb{P} V$ as the closure of the set of points where $Q$ is not continuous.

$Q$ is continuous on the dense open subset $\mathbb{P} V \backslash \mathbb{P}$ Ker $q_{V}$. It follows that

$$
M_{0}(Q)=\mathbb{P}\left(q_{V}(V)\right) .
$$

In particular, $M_{0}(Q)$ is a non-empty projective subspace of $\mathbb{P}(W)$ and $\operatorname{dim} M_{0}(Q)=$ $\operatorname{rank} q_{V}-1$.

It follows that an isomorphism $\mathbb{P} V \rightarrow \mathbb{P} W$ in the category of quasiprojective maps is the same as a projective isomorphism, i.e., a projective map induced by a linear isomorphism $V \rightarrow W$. And a quasiprojective map $Q: \mathbb{P} V \rightarrow \mathbb{P} W$ is an isomorphism onto its image iff $\operatorname{dim} M_{0}(Q)=\operatorname{dim} \mathbb{P} V$, in which case $Q(\mathbb{P} V)=$ $M_{0}(Q)$.

Also note that

$$
M_{0}\left(Q_{1} \circ Q_{2}\right)=M_{0}\left(Q_{1} \mid M_{0}\left(Q_{2}\right)\right)
$$

for any two composable quasiprojective maps $Q_{1}, Q_{2}$, by 4.3 .

4.5. Let us assume that $M_{0}(Q)$ consists of more than one point. Then $q_{V}$ has rank at least two, and is easily seen to be uniquely determined by $Q$ up to multiplication by a non-zero scalar. Furthermore

$$
M_{1}(Q)=\mathbb{P}\left(\operatorname{Ker} q_{V}\right),
$$


because if $\mathbb{R} q_{V}\left(e_{1}\right) \neq \mathbb{R} q_{V}\left(e_{2}\right)$ for two vectors $e_{1}, e_{2} \in V \backslash \operatorname{Ker} q_{V}$, then $Q(\mathbb{R}(x+$ $\left.\left.\varepsilon e_{i}\right)\right)=\mathbb{R} q_{0}\left(e_{i}\right)$ for $x \in \operatorname{Ker} q_{V}$ and every $\varepsilon \neq 0$.

The case that $M_{0}(Q)$ consists of one point only is quite different. There may be different sequences $q_{i}, V_{i}$ for the same quasi-projective map $Q$. And also different maps $q_{U}$ for vector subspaces $U$ of $V$. An extreme case is that the image of $Q$ consists of one point only. Still, in any case $M_{1}(Q)$ is a projective subspace of $\mathbb{P}(V)$, may be empty but is always a strict subspace of $\mathbb{P} V$, as we shall prove now.

4.6. Suppose $M_{0}(Q)$ consists of one point only. Put $M_{1}^{\prime}(Q)=\{x \in \mathbb{P} V ; Q(x) \neq$ $\left.M_{0}(Q)\right\}$ and let $U$ be the vector subspace of $V$ spanned by the lines corresponding to the points of $M_{1}^{\prime}(Q)$. Then $M_{1}(Q)=\overline{M_{1}^{\prime}(Q)}=\mathbb{P} U$.

Proof. If $M_{1}^{\prime}(Q)$ is empty, then $Q$ sends all of $\mathbb{P} V$ to $M_{0}(Q)$, hence $Q$ is continuous and $M_{1}(Q)=\mathbb{P}(\{0\})$ is empty as well. So suppose $M_{1}^{\prime}(Q)$ is not empty. The dense open subset $\mathbb{P} V \backslash \mathbb{P}$ Ker $q_{V}$ of $\mathbb{P} V$ has as $Q$-image the point $M_{0}(Q)$, so $M_{1}^{\prime}(Q) \subset M_{1}(Q)$. And $Q$ has value $M_{0}(Q)$ on the open subset $\mathbb{P} V \backslash \mathbb{P} U$ of $\mathbb{P} V$, so $M_{1}(Q) \subset \mathbb{P} U$. On the other hand, $q_{U}$ is a non-zero linear map, but $\mathbb{P}\left(q_{U}\right)$ does not map all of $\mathbb{P} U \backslash \mathbb{P}$ Ker $q_{U}$ to $M_{0}(Q)$, since otherwise $M_{1}^{\prime}(Q) \subset \mathbb{P}$ Ker $q_{U}$ and hence $U \subset \operatorname{Ker} q_{U}$. Thus $U$ contains a dense open subset $O$ with $\mathbb{P}(O) \subset M_{1}^{\prime}(Q)$. It follows that $\mathbb{P} U \subset \overline{M_{1}^{\prime}(Q)} \subset M_{1}(Q)$, by taking closures. This together with $M_{1}(Q) \subset \mathbb{P} U$ proves our claim.

4.7. The set of quasiprojective maps from $\mathbb{P}(V)$ to $\mathbb{P}(W)$ is closed with respect to the topology of pointwise convergence.

Proof. Let $\left(Q^{\alpha}\right)_{\alpha}$ be a convergent net in the set $\mathfrak{Q}$ of quasiprojective maps from $\mathbb{P}(V)$ to $\mathbb{P}(W)$. Let $U$ be a non-zero vector subspace of $V$ and let $q_{U}^{\alpha}$ be a non-zero linear map $U \rightarrow V$ representing $Q^{\alpha} \mid \mathbb{P} U$, so $\mathbb{P}\left(q_{U}^{\alpha}\right)=Q^{\alpha} \mid \mathbb{P}(U) \backslash$ $\mathbb{P}\left(\operatorname{Ker} q_{U}^{\alpha}\right)$. We choose norms on $V$ and $W$. The norm on $V$ induces a norm on $U$ and we thus have a norm $\|\cdot\|$ on the vector space $\mathcal{L}(U, W)$ of linear maps from $U$ to $W$. We may assume that $\left\|q_{U}^{\alpha}\right\|=1$ for every $\alpha$. So there is a convergent subnet $\left(q_{U}^{\beta}\right)_{\beta}$ of $\left(q_{U}^{\alpha}\right)_{\alpha}$ converging to a linear map $q_{U}: U \rightarrow W$ of norm 1. If $q_{U}(x) \neq 0$ then $q_{U}^{\beta}(x) \neq 0$ for $\beta$ large. For such $x$ we thus have $Q(\mathbb{R} x)=\lim Q^{\beta}(\mathbb{R} x)=$ $\lim \mathbb{R} q_{U}^{\beta}(x)=\mathbb{R} q_{U}(x)$. So $Q$ is quasiprojective.

4.8. If $\operatorname{dim} V \leq \operatorname{dim} W$, in particular if $V=W$, the set of quasiprojective maps $V \rightarrow W$ is the set of pointwise limits of projective maps $\mathbb{P}(V) \rightarrow \mathbb{P}(W)$.

Proof. By the last proposition it suffices to show that every quasiprojective map $Q: \mathbb{P}(V) \rightarrow \mathbb{P}(W)$ is a pointwise limit of projective maps. So let $q_{i}, V_{i}, i=$ $0, \ldots, r$, be as in 4.2. Extend every $q_{i}: V_{i} \rightarrow W$ to a linear map $V \rightarrow W$, also denoted $q_{i}$ and let $q_{r+1}: V \rightarrow W$ be an injective linear map. Define $q(\varepsilon)=$ 
$\sum_{j=0}^{r+1} \varepsilon^{j} q_{j}: V \rightarrow W$. We have $q(\varepsilon)=\varepsilon^{r+1} q^{\prime}\left(\frac{1}{\varepsilon}\right)$ with $q^{\prime}(s)=q_{r+1}+s q_{r}+\cdots+$ $s^{r+1} q_{0}$ for $\varepsilon \neq 0$, and $q^{\prime}(0)=q_{r+1}$ is injective, hence so is $q^{\prime}\left(\frac{1}{\varepsilon}\right)$ for $\varepsilon \gg 0$ and hence also $q(\varepsilon)$ for $\varepsilon \gg 0$. It follows that $q(\varepsilon)$ is injective except for at most a finite number of $\varepsilon$, by looking at the determinant of an appropriate submatrix in a matrix representation of $q(\varepsilon): V \rightarrow W$. Furthermore, $q(\varepsilon) \mid V_{i}=\varepsilon^{i} \sum_{j \geq i} \varepsilon^{j-i} q_{j}$, since $V_{i} \subset \bigcap_{\ell<i}$ Ker $q_{\ell}$, hence $\mathbb{P}(q(\varepsilon))$ converges pointwise to $\mathbb{P}\left(q_{i}\right)=Q$ on $\mathbb{P}\left(V_{i}\right)$ for $\varepsilon \rightarrow 0$.

4.9. The set $Q(V)$ of quasiprojective maps of $\mathbb{P}(V)$ to itself is a semigroup and a compact topological space with respect to the topology of pointwise convergence. Right translation in $Q(V)$ is continuous, but left translation is not, in general. But if $Q \in Q(V)$ is a continuous map $\mathbb{P}(V) \rightarrow \mathbb{P}(V)$, in particular, if $Q$ is a projective isomorphism $\mathbb{P}(V) \rightarrow \mathbb{P}(V)$, then left translation in $Q(V)$ with $Q$ is continuous, i.e., the map $Q(V) \rightarrow Q(V)$, with $R \longmapsto Q \circ R$, is continuous.

Note that contrary to what one might think after 4.8 , the topological space $Q(V)$ is not metrisable, in general. E.g., if $\operatorname{dim} V=2$, the quasiprojective not projective maps $\mathbb{P}^{1} \rightarrow \mathbb{P}^{1}$ are exactly the maps which are constant on the complement of one point. In the set of these maps with the topology of pointwise convergence no point has a countable neighborhood base, hence this space is not metrisable.

4.10. A quasiprojective map $b$ is called a contraction if $M_{0}(b)$ consists of one point only. The notion of contraction is closely related to that of a contractive sequence of linear maps, see [AMS1, p. 6ff.] and [GM, Lemma $6.5 \mathrm{f}$.] Let $H$ be a subsemigroup of $G L(V)$ and let $\bar{H}$ be the closure of $H$ in the semigroup $Q(\mathbb{P})$ of quasiprojective selfmaps of $\mathbb{P}=\mathbb{P}(V)$. If $H$ contains a proximal element $h$, then $\bar{H}$ contains a contraction. In fact, the sequence $h^{n}, n \in \mathbb{N}$, contains a subsequence which converges in $Q(\mathbb{P})$, and every limit $b$ of a convergent subsequence of $h^{n}$, $n \in \mathbb{N}$, is a contraction.

The converse is not true. Even if $\bar{H} \backslash H$ consists of contractions, it does not follow that $H$ contains a proximal element. A simple counterexample - which kills many hopes - is the following. Let $H$ be the subsemigroup of $S L_{2}(\mathbb{Z})$ of upper triangular unipotent matrices with upper right hand corner entry in $\mathbb{N}$. Let $b$ be the quasiprojective map $\mathbb{P}^{1} \rightarrow \mathbb{P}^{1}$ whose image is $\mathbb{P}\left(e_{1}\right)$, where $e_{1}$ is the first standard basis vector of $\mathbb{R}^{2}$. So $b$ is a contraction. Then $\bar{H}$ consists of $H$ and $b$. But $H$ contains no proximal element.

Also note that this semigroup $H$ consists of non-negative matrices and maps the open cone $P=\left\{(x, y) \in \mathbb{R}^{2} ; x>0\right.$ and $\left.y>0\right\}$ into itself. Still, in contrast to Perron's theorem, $H$ does not contain a proximal element. 
The problem here is related to remark 2.2. In more detail, let $h_{n}$ be a sequence in $G L(V)$ which converges to a contraction $b \in Q(\mathbb{P})$. By passing to a subsequence we may assume that $\frac{h_{n}}{\left\|h_{n}\right\|}$ converges to a linear map $h \in \operatorname{End}(V)$. Here we suppose that a norm $\|\cdot\|$ on $V$ was chosen. Then $h$ has rank 1 , since $\mathbb{P} h$ : $\mathbb{P} V \backslash \mathbb{P}$ Ker $h \rightarrow \mathbb{P}$ and $b$ coincide on the open dense subset $M_{0}(b) \cap(\mathbb{P} V \backslash \mathbb{P K e r} h)$ of $\mathbb{P}$. It follows that if $\operatorname{Im}(h) \not \subset \operatorname{Ker}(h)$, then $H$ is proximal, by 2.2 , and hence so are $h_{n}$ for $n \gg 0$, by 2.3. But if $\operatorname{Im}(h) \subset \operatorname{Ker}(h)$ this may or may not be the case. And knowing $b$ is not enough to decide if $\operatorname{Im}(h) \not \subset \operatorname{Ker}(h)$, as the example above shows. In the proof of theorem 5.1 irreducibility of the semigroup $H$ on $V$ lets us find an element $h$ with $\operatorname{Im}(h) \not \subset \operatorname{Ker}(h)$, see the second paragraph of the proof of 5.1 .

Let us look at these questions from a different point of view, namely moduli of eigenvalues vs. singular values.

4.11. The spectral radius $\rho(g)$ of $g \in \operatorname{End}(V)$ is by definition the maximal modulus of the eigenvalues of $g$. Let $\lambda_{1}, \ldots, \lambda_{n}$ be the eigenvalues of $g$, each written with its algebraic multiplicity and ordered such that $\left|\lambda_{1}\right| \geq\left|\lambda_{2}\right| \geq \cdots \geq\left|\lambda_{n}\right|$. So $\rho(g)=\left|\lambda_{1}\right|$. Let $\Lambda^{r} g: \Lambda^{r} V \rightarrow \Lambda^{r} V$ be the linear endomorphism of the exterior power $\Lambda^{r} V$ induced by $g$, i.e., such that $\Lambda^{r} g\left(v_{1} \wedge \cdots \wedge v_{r}\right)=g v_{1} \wedge \cdots \wedge g v_{r}$. Then $\rho\left(\Lambda^{r} g\right)=\left|\lambda_{1} \ldots \lambda_{r}\right|$. Hence $g$ is proximal iff $\rho(g)>0$ and $\rho\left(\Lambda^{2} g\right) / \rho(g)^{2}<1$.

4.12. Suppose we have a norm $\|\cdot\|$ on the vector space $V$. Let $\|\cdot\|$ also denote the associated operator norm on $\operatorname{End}(V)$. Then $\lim _{n \rightarrow \infty} \sqrt[n]{\left\|g^{n}\right\|}=\rho(g)$. Thus $g$ is proximal iff $g \neq 0$ and

$$
\lim _{n \rightarrow \infty} \sqrt[n]{\frac{\left\|\Lambda^{2} g^{n}\right\|}{\left\|g^{n}\right\|^{2}}}<1,
$$

or equivalently if $g \neq 0$ and

$$
\lim \frac{1}{n} \log \left\|\Lambda^{2} g^{n}\right\|<2 \lim _{n \rightarrow \infty} \frac{1}{n} \log \left\|g^{n}\right\| .
$$

4.13. Note that $\lim _{n \rightarrow \infty}\left\|\Lambda^{2} g^{n}\right\| /\left\|g^{n}\right\|^{2}=0$ does not imply that $g$ is proximal. E.g., if $g \in S L_{2}(\mathbb{R})$ is unipotent. Here $\Lambda^{2} g=$ id and $\left\|g^{n}\right\|$ grows only polynomially. For proximality we need exponential growth.

4.14. Suppose we have a norm on $V$ and $h \in \operatorname{End}(V)$ has large operator norm and there is an $h$-invariant hyperplane $H$ on which the norm of $h \mid H$ is small. These properties of $h$ do not imply that $h$ is proximal, e.g., if $h$ is a multiple of a unipotent upper triangular $2 \times 2$-matrix. Thus information about the singular values of $h$, equivalently, knowledge of $\|h\|,\left\|\Lambda^{2} h\right\|, \ldots$, does not imply proximality. 


\section{The TheOrem of Gol'DSheID-Margulis}

A subsemigroup $\Gamma$ of $G L(V)$ is called strongly irreducible if there is no finite union $\bigcup_{i} V_{i}$ of vector subspaces $V_{i}$ of $V$ with $0<V_{i}<V$, which is invariant under $\Gamma$. Recall that the Zariski closure of a subsemigroup $\Gamma$ of $G L(V)$ is an algebraic subgroup. If $G$ is the closure of $\Gamma$, then $\Gamma$ is strongly irreducible iff the connected component $G^{0}$ of the identity in $G$ is irreducible. Here one can take the connected component of $G$ with respect to the Euclidean topology or with respect to the Zariski topology, the criterion will be the same.

Theorem 5.1 (Gol'dsheid-Margulis). Let $G$ be a strongly irreducible subgroup of $G L(V)$ which contains a proximal linear map. Then every Zariski dense subsemigroup $\Gamma$ of $G$ is strongly irreducible and contains a proximal element.

Proof. The first claim follows from what was said above. The point is to show the second claim. We may and will assume that $G$ is the Zariski closure of $\Gamma$. Let $\mathbb{P}(\Gamma)$ be the semigroup $\{\mathbb{P}(\gamma), \gamma \in \Gamma\}$ of projective selfmaps of $\mathbb{P}(V)$. Let $H$ be the closure of $\mathbb{P}(\Gamma)$ with respect to the pointwise topology of $\mathbb{P}(V)$. So $H$ is a closed subsemigroup of $Q(V)$. We shall prove that $H$ contains an element $h$ with $M_{0}(h)$ consisting of one point only.

This implies our claim by the following argument. Let $e_{1}, \ldots, e_{n}$ be a basis of $V$ with $\mathbb{P}\left(e_{i}\right) \notin M_{1}(Q)$ for $i=1, \ldots, n$. Let $h_{j}, j \in \mathbb{N}$, be a sequence of elements of $\Gamma$ such that $\mathbb{P}\left(h_{j}\right) \mathbb{P}\left(e_{i}\right)$ converges to $M_{0}(h)$ for $j \rightarrow \infty$ and every $i=1, \ldots, n$. Choose a norm $\|\cdot\|$ on $V$ and let $v$ be a vector of norm 1 such that $\mathbb{P}(v)=M_{0}(h)$. Then, for every $i$, we may assume that $\frac{h_{j}\left(e_{i}\right)}{\left\|h_{j}\left(e_{i}\right)\right\|}$ converges to $+v$ or to $-v$. It follows that the sequence of linear maps $\frac{h_{j}}{\left\|h_{j}\right\|}$ converges in $\operatorname{End}(V)$ to a linear map $\widetilde{h}$ of rank one with image $\operatorname{Im}(\widetilde{h})=\mathbb{R} v$. If $v \notin \operatorname{Ker} \widetilde{h}$, then $\widetilde{h}$ is proximal by 2.2 , hence so is $\frac{h_{j}}{\left\|h_{j}\right\|}$ for $j$ large by 2.3 and hence so is $h_{j} \in \Gamma$. If $v \in \operatorname{Ker} \widetilde{h}$, take an element $g \in \Gamma$ such that $g v \notin \operatorname{Ker} \widetilde{h}$, which is possible since $\Gamma$ is irreducible. Then $g \cdot \frac{h_{j}}{\left\|h_{j}\right\|}$ converges to $g \widetilde{h}$, which is of rank one and $\operatorname{Im}(g \widetilde{h})=g \mathbb{R} v \not \subset \operatorname{Ker} \widetilde{h}=\operatorname{Ker} g \widetilde{h}$, hence $g h_{j} \in \Gamma$ is proximal for $j$ large.

So suppose there is no $h \in H$ with $M_{0}(h)$ consisting of one point only. Let $d$ be the minimal dimension of spaces $M_{0}(h), h \in H$. So $d>0$.

Lemma 5.2. Suppose $b \in H$ and $\operatorname{dim} M_{0}(b)=d$. Then for every $h \in H$ we have $\operatorname{dim} M_{0}(h b)=d$ and $h \mid M_{0}(b)$ is a projective isomorphism from $M_{0}(b)$ onto its image and this image is $M_{0}(h b)$.

Proof. $M_{0}(h b)=M_{0}\left(h \mid M_{0}(b)\right)$ has dimension $\leq d=\operatorname{dim} M_{0}(b)$, by 4.4 , so $d=$ $M_{0}\left(h \mid M_{0}(b)\right)$ by minimality of $d$, which implies all our claims by 4.4 . 
Lemma 5.3. Suppose $b \in H$ and $\operatorname{dim} M_{0}(b)=d$. Then for every $h \in H$ we have

$$
M_{0}(b) \subset M_{1}(h) \text { or } M_{0}(b) \cap M_{1}(h)=\emptyset .
$$

Proof. If $h_{V}$ represents $h$ on $V$, we have $M_{1}(h)=\mathbb{P}$ Ker $h_{V}$, by 4.5 , since $\operatorname{dim} M_{0}(h) \geq d>0$. Hence if $M_{1}(h) \cap M_{0}(b) \neq \emptyset$ but $M_{0}(b) \not \subset M_{1}(h)$, then $h_{V} \mid W$ is non-zero but not injective. Here $W$ is the vector subspace of $V$ such that $M_{0}(b)=\mathbb{P} W$. Suppose $b_{V}$ represents $b$ on $V$, then $\operatorname{Im} b_{V}=W$ and $\left(h_{V} \mid W\right) \circ b_{V}$ represents $h \circ b$ on $V$ and hence $M_{0}(h \circ b)=\mathbb{P} \operatorname{Im}\left(\left(h_{V} \mid W\right) \circ b_{V}\right)$ has dimension $<\operatorname{dim} M_{0}(b)=d$, contradicting the minimality of $d$.

Lemma 5.4. There is an element $b \in H$ such that $\operatorname{dim} M_{0}(b)=d$ and $M_{0}(b) \cap$ $M_{1}(b)=\emptyset$.

Proof. Recall that if $g \in P G L(V)$ and $q$ is quasiprojective then

$$
\begin{aligned}
& M_{0}(g q)=g M_{0}(q), \\
& M_{0}(q g)=M_{0}(q), \\
& M_{1}(g q)=M_{1}(q), \\
& M_{1}(q g)=g^{-1} M_{1}(q) .
\end{aligned}
$$

For $b \in H$ with $\operatorname{dim} M_{0}(b)=d$ look at the set of $\gamma \circ b, \gamma \in \Gamma$. Then $M_{0}(\gamma \circ$ $b)=\gamma M_{0}(b)$ has either empty intersection with $M_{1}(\gamma b)=M_{1}(b)$ for one $\gamma \in \Gamma$ or, by the previous lemma, $\gamma M_{0}(b) \subset M_{1}(b)$ for all $\gamma \in \Gamma$, which contradicts irreducibility of $\Gamma$. Recall that $M_{1}(b) \neq \mathbb{P} V$.

We are now close to the key point of the proof. It has a very transparent geometry, as follows. Lemma 5.4 will provide us with a linear subspace $W$ of $V$, namely $\mathbb{P} W=M_{0}(b)$, and a quasiprojective map $b \in H$ with the following properties. 1) $b$ is a retraction of $P$ onto $\mathbb{P} W$ outside of some projective subspace (our $M_{1}(b)$ ) which does not intersect $\mathbb{P} W$. 2) Every map $\varphi \in H$ with $\varphi(\mathbb{P} w) \subset$ $\mathbb{P} W$ induces a projective isomorphism $\beta(\varphi)$ of $\mathbb{P} W$. This is due to the minimality of $\operatorname{dim} \mathbb{P} W$, technically speaking, it is a consequence of lemma 5.2.

We will set $\Phi=\{\varphi \in H ; \varphi(\mathbb{P} W) \subset \mathbb{P} W\}$. It will follow that the compact subsemigroup $\beta(\Phi)$ of $P G L(\mathbb{P} W)$ is actually a compact subgroup and hence a real algebraic subgroup of $P G L(\mathbb{P} W)$, by a theorem of Chevalley.

Now for a certain Zariski open subset of $G$, the set $G_{b}$ below, the composition $b \circ g$ of $g$ with the retraction $b$ induces a projective isomorphism $\beta(b \circ g)$ of $\mathbb{P} W$. We just have to make sure that $g \mathbb{P} W$ does not intersect the singular set $M_{1}(b)$ of the retraction $b$. But for the Zariski dense subset $\Gamma \cap G_{b}$ the image $\beta(b \circ g)$ lies in $\Phi$, hence so does $\beta(b \circ g)$ for every $g \in G_{b}$. But this will be easily seen to contradict the hypothesis that $G$ contains a proximal element and thus a sequence 
converging to a quasiprojective map with $M_{0}$ a one point set. Remember that we assumed that $d=\operatorname{dim} \mathbb{P} W>0$.

Here are the details now. Let $b \in H$ be as in lemma 5.4. Let $W$ be the vector subspace of $V$ with $\mathbb{P} W=M_{0}(b)$. Then every linear map $b_{V}$ representing $b$ on $V$ is a surjection from $V$ onto $W$. And $\mathbb{P}$ Ker $b_{V}=M_{1}(b)$, by 4.5, so $b_{V}$ restricts on $W$ to a linear automorphism of $W$. More generally, set

$$
\Phi=\{\varphi \in H ; \varphi(\mathbb{P} W) \subset \mathbb{P} W\} .
$$

Clearly, $b \in \Phi$ and $\Phi$ is a compact subsemigroup of $H$. For $\varphi \in \Phi$ let $\beta(\varphi)$ be the quasiprojective map $\mathbb{P} W \rightarrow \mathbb{P} W$ induced by $\varphi$. Then $\beta(\varphi)$ is actually a projective automorphism of $\mathbb{P} W$, by lemma 5.2. Thus $\beta$ gives a homomorphism $\Phi \rightarrow P G L(\mathbb{P} W)$. Its image $\beta(\Phi)$ is thus a compact subsemigroup of $P G L(\mathbb{P} W)$.

We now use the following two facts.

- Every compact subsemigroup of a topological group is a subgroup.

- Every compact subgroup of a real algebraic group is Zariski closed.

The second one of these facts is a non-trivial theorem due to Chevalley. The first one is quite elementary and we give a proof now. Let $H$ be a compact subsemigroup of the topological group $G$. Let $g$ be an element of $H$. We only have to prove that $g^{-1} \in H$. The sequence $\left(g^{n}\right)_{n \in \mathbb{N}}$ has a convergent subnet, say $\left(g^{n_{i}}\right)_{i \in I}$ converges to $h \in H$. For every $m \in \mathbb{N}$ the net $\left(g^{n_{i}-m}\right)_{i \in I}$ converges to $g^{-m} h$ which is an element of $H$, since $n_{i}-m>0$ for $i$ sufficiently large. It follows that the net $\left(g^{-n_{i}-1} h\right)_{i \in I}$ consists of elements of $H$. This net converges to $g^{-1}$, which is hence also in $H$.

It follows from the two facts stated above that $\beta(\Phi)$ is a Zariski closed compact subgroup of $P G L(\mathbb{P} W)$.

Again, let $b$ be as in lemma 5.4. Set

$$
G_{b}=\left\{g \in G ; M_{1}(b) \cap g M_{0}(b)=\emptyset\right\} .
$$

Then $G_{b}$ is a non-empty Zariski open subset of $G$ containing the identity element. We claim that $b g$ induces a projective isomorphism of $\mathbb{P} W$. The proof is similar as above: Let $b_{V}$ be the linear map representing $b$ on $V$. We know that $\operatorname{Im} b_{V}=W$ and $\mathbb{P K e r} b_{V}=M_{1}(b)$. Thus $\mathbb{P I m} b_{V} \circ g=\mathbb{P} W=M_{0}(b)$ and $\mathbb{P K e r} b_{V} \circ g=$ $g^{-1} M_{1}(b)$ and these two subspaces of $\mathbb{P} W$ do not intersect, by definition of $G_{b}$. So $\mathbb{P}\left(b_{V} \circ g\right)=b g \mid \mathbb{P} V \backslash \mathbb{P K e r} b_{V} \circ g$, hence $b g$ induces a projective isomorphism of $\mathbb{P} W$ onto itself.

For $g \in G_{b}$ let $\beta(b g) \in P G L(\mathbb{P} W)$ be the projective map of $\mathbb{P} W$ to itself induced by $b g$. We thus have a morphism $G_{b} \rightarrow P G L(\mathbb{P} W), g \longmapsto \beta(b g)$, of real algebraic varieties which maps the Zariski dense subset $\Gamma \cap G_{b}$ to the Zariski closed compact subgroup $\beta(\Phi)$, hence so does $G_{b}$, i.e., $\beta(b g)$ is contained in the 
compact subgroup $\beta(\Phi)$ for every $g \in G_{b}$. But this contradicts the hypothesis that $G$ contains a proximal element, as we will prove now.

Let $G^{0}$ be the connected component of the identity in $G$, with respect to the Zariski topology.

$G$ contains a proximal element, hence so does $G^{0}$, since $G / G^{0}$ is finite and every positive power of a proximal map is proximal. For a proximal linear map $h$ let $L_{h}^{+}$ be the eigenspace of the dominant eigenvalue and let $H_{h}^{-}$be the complementary $h$-invariant hyperplane. We claim that there is a proximal element $h \in G^{0}$ such that $L_{h}^{+} \not \subset M_{1}(b)$ and $M_{0}(b) \not \subset H_{h}^{-}$. If $h$ is proximal, then so are its conjugates $g h g^{-1}$, and $L_{g h g^{-1}}^{+}=g L^{+}$and $H_{g h g^{-1}}^{-}=g H_{h}^{-}$. So for a fixed proximal element $h \in G^{0}$ the set of $g \in G^{0}$ such that $g L_{h}^{+} \not \subset M_{1}(b)$ and $g^{-1} M_{0}(b) \not \subset H_{h}^{-}$is Zariski open and not empty, since $V$ is an irreducible $G^{0}$-module. The set

$$
P=\left\{g \in G^{0} ; g \text { proximal, } L_{g}^{+} \not \subset M_{1}(b), M_{0}(b) \not \subset H_{g}^{-}\right\}
$$

is thus not empty. Also, $P$ is open in $G^{0}$ with respect to the Euclidean topology. It follows that $P \cap G_{b}$ is not empty, since $G_{b} \cap G^{0}$ is Zariski open and not empty in the irreducible variety $G^{0}$. Now for $g \in P \cap G_{b}$ the sequence $g^{n} \mathbb{P} W$ converges to $\mathbb{P} L_{g}^{+}$, uniformly, hence $g^{n} \in G_{b}$ for $n$ large. It follows that the sequence of quasiprojective maps $b g^{n}(\mathbb{P} W)$, represented by $b_{V} g^{n} \mid W$, converges to the map which sends $\mathbb{P} W$ to the one point $b\left(\mathbb{P} L_{g}^{+}\right)$. So the sequence $P\left(b g^{n}\right)$ cannot be contained in a compact subset of $P G L(\mathbb{P} W)$.

The quasiprojective maps in $H$ are a method of bookkeeping of properties of limits of sequences of maps in $\Gamma$. We used this here to prove that $\Gamma$ contains a proximal map if the minimal dimension of $M_{0}(h), h \in H$, is zero and the representation is irreducible.

Note that we used the hypothesis that $G$ contains a proximal element only at the very end of the proof of theorem 5.1. Thus the same proof gives the following result. Let $M(g)$ be the sum of the algebraic multiplicities of all eigenvalues $\lambda$ of $g$ of maximal modulus. Thus $g$ is proximal iff $M(g)=1$.

Theorem 5.5. Let $G$ be a strongly irreducible subgroup of $G L(V)$ and let $M$ be the minimum of the numbers $M(g), g \in G$. Then every Zariski dense subsemigroup $\Gamma$ of $G$ contains an element $\gamma$ with $M(\gamma)=M$.

The proof of theorem 5.1 given here is taken from [GM, §6]. The "elementary proof" in [GM, §2,3] for the case that $G=G L(V)$ contains a gap. Lemma 2.17 in $[\mathrm{GM}]$, though true for projective space, is not true for the space of flags.

Other proofs of theorem 5.1 have been given. The proof in $[\mathrm{P}]$ avoids using the topology of $Q(V)$ and uses only limiting maps in $\operatorname{End}(V)$. The proof in [BL] is 
based on probabilistic results in [GR]. The argument of [GM], however, is very basic and simple and we thus thought it useful to give a detailed account of it.

\section{Generalizations And Applichtions}

6.1. One might ask if a similar result as theorem 5.1 holds for non-archimedian fields. The definition of proximality is literally the same for local fields as for $\mathbb{R}$. The proof certainly does not work for non-archimedian fields since it uses at a crucial point the theorem that a compact subgroup of an affine algebraic group over $\mathbb{R}$ is Zariski closed. Thus the question above has a negative answer for a trivial reason. For non-archimedian fields $k$ there are compact Zariski dense subgroups, e.g. $S L_{n}(\mathcal{O})$ in $S L_{n}(k)$, where $\mathcal{O}$ is the maximal compact subring of $k$. But also the following non-trivial question has a negative answer. Suppose $\Gamma$ is a Zariski dense subgroup of a strongly irreducible Zariski closed subgroup $G$ of $G L(V)$. Suppose $G$ contains a proximal element and $\Gamma$ is not contained in a compact subgroup of $G$. Does then $\Gamma$ contain a proximal element? The answer is again no. A counterexample is due to $\mathrm{G}$. Margulis and published in $[\mathrm{Pl}]$.

6.2. In [AMS1] we prove that under the hypotheses of theorem $5.1 \Gamma$ not only contains one proximal element, but a rich supply. The step from one proximal to many works also for other local fields, we thus isolate this result for other applications, see [S].

Theorem 6.3. Let $\Gamma$ be a subsemigroup of the linear group $G L(V)$ of a finite dimensional vector space $V$ over a local field $k$. Suppose $\Gamma$ is strongly irreducible and that $\Gamma$ contains a proximal element. Then there is a subset $M$ of $\Gamma$ with card $(M) \leq(\operatorname{dim}(V))^{2}$ such that for every $g \in G L(V)$ there is a $\gamma \in M$ for which $\gamma^{n} g$ is proximal for every natural number $n$.

The proof is the same as for the $k=\mathbb{R}$, see [AMS1, 4.1]. The result is actually true for a quantitative version of proximality, taking into account the quotient of the modulus of the dominant eigenvalue of $g$ divided by the norm of $g$ on the complementary $g$-invariant hyperplane $H^{-}$and the distance between $L^{+}$and $H^{-}$.

6.3 implies easily that under the hypotheses of 6.3 the set of proximal elements of $\Gamma$ is Zariski dense. One can furthermore show that the set of elements $\gamma \in \Gamma$ such that both $\gamma$ and $\gamma^{-1}$ are proximal is Zariski dense, see [AMS1, 4.11]. This is important for applications of the ping-pong lemma.

6.4. Here is a nice and useful application of theorem 5.1. Now everything is over $\mathbb{R}$. Let $G$ be a reductive subgroup of $G L(V)$ defined over $\mathbb{R}$. Let Ad be the adjoint representation of $G$ on its Lie algebra $\mathfrak{g}$. We assume that $G$ is not compact. An element $g \in G$ is called $\mathbb{R}$-regular if the number of eigenvalues, counted with multiplicities, of modulus 1 is minimum possible. It is known that 
every $\mathbb{R}$-regular element is semisimple [PRa] and it is easy to see that an element of $S L_{n}(\mathbb{R})$ is $\mathbb{R}$-regular iff all its eigenvalues in the natural $n$-dimensional representation are real and their moduli are distinct. The first two parts of the following theorem were also proved in $[\mathrm{BL}]$ and $[\mathrm{P}]$.

Theorem 6.5. Let $\Gamma$ be a Zariski dense subsemigroup of the reductive group $G$. Then $\Gamma$ contains an $\mathbb{R}$-regular element. In fact, the set of $\mathbb{R}$-regular elements in $\Gamma$ is Zariski dense. There is a finite subset $M$ of $\Gamma$ such that for every $g \in G$ there is at least one $\gamma \in M$ such that $\gamma^{n} g$ is $\mathbb{R}$-regular for every natural number $n$.

The proof in $[\mathrm{AMS} 1,6.8]$ uses the following representation. Let $\mathfrak{u}$ be the Lie algebra of the unipotent radical of a minimal parabolic subgroup of $G$ and let $k$ be its dimension. Let $V$ be the smallest $G$-submodule of $\wedge^{k} \mathfrak{g}$ containing the line $\wedge^{k} \mathfrak{u}$. Then $V$ is a strongly irreducible representation of $G$, with highest weight sum of the positive relative roots.

Let $\rho$ be the corresponding representation. An element $g \in G$ is $\mathbb{R}$-regular iff $\rho(g)$ is proximal. Since such elements exist in $G$, the theorem follows.

Here is another description of the elements for which $\rho(g)$ is proximal. Recall that every element $g \in G L(V)$ has a multiplicative Jordan decomposition $g=$ $s \cdot u=u \cdot s$ uniquely determined by the conditions that $s$ is semisimple and $u$ is unipotent and $s$ and $u$ commute. Every semisimple element $s \in G L(V)$ has a polar decomposition $s=p \cdot k=k \cdot p$ uniquely determined by the conditions that $p$ and $k$ are semisimple, the eigenvalues of $p$ are positive real, the eigenvalues of $k$ have modulus one, and $p$ and $k$ commute. The elements $s=s(g)$ and $u=u(g)$ are called the semisimple and unipotent part of $g$, and $p$ and $k$ are called the polar and compact part of $s$ or of $g$ if $s=s(g)$.

Now, $g$ is $\mathbb{R}$-regular iff the polar part $\operatorname{pol}(g)$ of $g$ is regular, i.e., its centralizer has maximal dimension or equivalently $\operatorname{pol}(g)$ is conjugate to an inner point of a Weyl chamber of some maximal $\mathbb{R}$-split torus of $G$. For results on the asymptotic behavior of $\operatorname{pol}(\gamma), \gamma \in \Gamma$, their limit directions and the number of elements below a given upper bound, see the work of Benoist and Quint.

6.6. For our work on properly discontinuous and crystallographic affine groups we needed a more precise result giving also information about the compact part of elements of $\Gamma$ : They should generate a dense subgroup of the maximal compact subgroup of a maximal (non-split) torus in $G$. Such results are proved in the papers [PR1, PR2] of Prasad and Rapinchuk. They use algebraic methods. These results do not seem to be attainable by the methods presented here. 


\section{ApPENDix}

The cross ratio For any four points $a, b, c, d$ on the real line $\mathbb{R}$ we define their cross ratio by

$$
[a, b, c, d]=\frac{c-a}{b-a} \cdot \frac{d-b}{d-c} .
$$

Here we assume $a \neq b$ and $c \neq d$ in order to avoid that we have to calculate with $\infty$. It is well known and easy to compute that for every real projective automorphism $f$ of $\mathbb{P}_{\mathbb{R}}^{1}=\mathbb{R} \cup\{\infty\}$ we have

$$
[f(a), f(b), f(c), f(d)]=[a, b, c, d],
$$

where we suppose for convenience, that none of the points $a, b, c, d$ is mapped to $\infty$ by $f$. Thus, if we have four points $A, B, C, D$ with $A \neq B$ and $C \neq D$ on an affine line $L$ in a real vector space $V$, their cross ratio is well defined by

$$
[A, B, C, D]=[\lambda(A), \lambda(B), \lambda(C), \lambda(D)],
$$

if $\lambda: L \rightarrow \mathbb{R}$ is an affine coordinate system on $L$. By denoting $\lambda(B)-\lambda(A)$ by $A B$ etc. we obtain

$$
[A, B, C, D]=\frac{A C}{A B} \cdot \frac{B D}{C D} \text {. }
$$

If furthermore the four points $A, B, C, D$ are located in this order on $L$, we can think of $A C$ etc. as just the distance between $A$ and $C$ with respect to a given norm on $V$. Then their cross ratio is $\geq 1$ and $>1$ if $B \neq C$.

The Hilbert metric The Hilbert metric $d_{X}$ of a bounded convex open subset $X$ of $\mathbb{R}^{n}$ is defined as follows: For any two different points $x, y$ of $X$ let $x^{\prime}$ and $y^{\prime}$ be the points of $\partial X$ on the line $\ell$ through $x$ and $y$ such that $x^{\prime}, x, y, y^{\prime}$ are located in this order on $\ell$. Then the Hilbert metric is defined by

$$
d_{X}(x, y)=\log \left[x^{\prime}, x, y, y^{\prime}\right]
$$

\section{Properties of the Hilbert metric:}

a) (Monotonicity) If $X \subset Y$ then $d_{X}(x, y) \geq d_{Y}(x, y)$ for $x, y \in X$, since the term $\frac{x^{\prime} y}{x^{\prime} x}=\frac{x^{\prime} x+x y}{x^{\prime} x}=1+\frac{x y}{x^{\prime} x}$ in the cross ratio decreases for $Y \supset X$ to $1+\frac{x y}{x^{\prime \prime} x}$ if $x^{\prime \prime}$ is the point of $\partial Y$ on the line $\ell$ through $x$ and $y$, and similarly for the other factor $\frac{x y^{\prime}}{y y^{\prime}}$. And since $x^{\prime} x^{\prime \prime}$ is bounded below if $\bar{X} \subset Y$, we have:

b) if $\bar{X} \subset Y$, then there is a number $\delta<1$ such that

$$
d_{Y}(x, y) \leq \delta \cdot d_{X}(x, y) \text { for every } x, y \in X .
$$

c) (Additivity) If $z \in[x, y]$ then

$$
d_{X}(x, y)=d_{X}(x, z)+d_{X}(z, y),
$$

by direct calculation. 
d) (Triangle inequality) Given $x, y, z$ in $X$, the convex set $X$ contains the quadrilateral $Y$ whose diagonals are the maximal segments $\left[x^{\prime}, y^{\prime}\right]$ and $\left[y^{\prime \prime}, z^{\prime \prime}\right]$ in $X$ containing $[x, y]$ and $[y, z]$, respectively. Let $\left[x^{\prime \prime \prime}, z^{\prime \prime \prime}\right]$ be the maximal segment in $Y$ containing $[x, z]$ and let $p$ be the point of intersection of the lines containing $\left[x^{\prime}, y^{\prime \prime}\right]$ and $\left[y^{\prime}, z^{\prime \prime}\right]$. If these lines are parallel we take $p$ to be infinity. Projection from $p$ sends $y$ to a point, say $w$, in $[x, z]$. Since projection between lines preserves cross ratios, we see that $\left[x^{\prime}, x, y, y^{\prime}\right]=\left[x^{\prime \prime \prime}, x, w, z^{\prime \prime \prime}\right]$ and hence

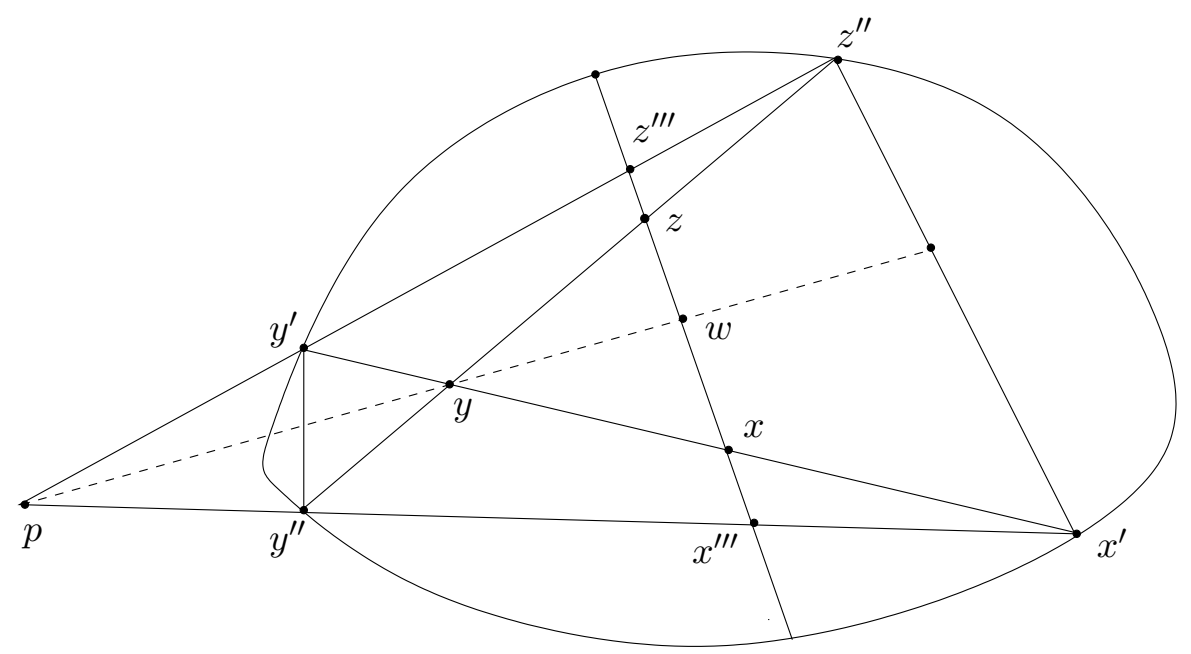

Figure 1. Triangle inequality.

and thus

$$
\begin{aligned}
& d_{X}(x, w) & \leq d_{Y}(x, w) & =d_{X}(x, y) \\
\text { and } & d_{X}(w, z) & \leq d_{Y}(w, z) & =d_{X}(y, z)
\end{aligned}
$$

$$
d_{X}(x, z)=d_{X}(x, w)+d_{X}(w, z) \leq d_{X}(x, y)+d_{X}(y, z) .
$$

e) The Hilbert metric is complete and induces the topology of $X$, as is easy to see.

\section{REFERENCES}

[A] Abels, H., Properly discontinuous groups of affine transformations: a survey, Geom. Dedicata, 87, (2001), 309-333

[AMS1] Abels, H., G.A. Margulis and G.A. Soifer, Semigroups containing proximal linear maps, Israel J. of Math., 91, (1995), 1-30

[AMS2] Abels, H., G.A. Margulis and G.A. Soifer, Properly discontinuous groups of affine transformations with orthogonal linear part, Comptes Rendus Acad. Sc. Paris, 324 I, (1997), 253-258 
[AMS3] Abels, H., G.A. Margulis and G.A. Soifer, On the Zariski closure of the linear part of a properly discontinuous group of affine transformations, J. Differential Geom., 60, (2002), 315-344

[AMS4] Abels, H., G.A. Margulis and G.A. Soifer, The Auslander conjecture for groups leaving a form of signature $(n-2,2)$ invariant, Israel J. Math., 148, (2005), 11-22

[AMS5] Abels, H., G.A. Margulis and G.A. Soifer, The linear part of an affine group acting proper discontinuous and leaving quadratic form invariant, SFB 701-Preprint 06-002

[B1] Benoist, Y., Automorphismes des cônes convexes, Invent. Math., 141, (2000), 149-193

[B2] Benoist, Y., Propriétés asymptotiques des groupes linéaires. II, Adv. Stud. Pure Math., 26, (2000), 33-48

[B3] Benoist, Y., Convexes divisibles. I, Tata Inst. Fund. Res., Mumbai, (2004), 339-374

[B4] Benoist, Y., Convexes divisibles II, Duke Math. J., 120, (2003), 97-120

[B5] Benoist, Y., Convexes divisibles. III, Ann. Sci. École Norm. Sup. (4), 38, (2005), 793832

[BG] Breuillard, E. and T. Gelander, On dense free subgroups of Lie groups, J. Algebra, 261, (2003), 448-467

[BL] Benoist, Y. and F. Labourie, Sur les difféomorphismes d'Anosov affines à feuilletages stable et instable différentiables, Invent. Math., 111, (1993), 285-308

[BP] Berman, A. and R.K. Plemmons, Nonnegative matrices in the mathematical sciences, Revised reprint of the 1979 original, Classics in Applied Mathematics, 9. SIAM, Philadelphia, PA, 1994

[Fu] Furstenberg, H., Boundary theory and stochastic processes on homogeneous spaces, Harmonic analysis on homogeneous spaces, Proc. Sympos. Pure Math., Vol. XXVI, (1972), Amer. Math. Soc., Providence, R.I., (1973), 193-229

[G] Gantmacher, F.R., The theory of matrices, Translated from the Russian by K. A. Hirsch, Reprint of the 1959 translation, AMS Chelsea Publishing, Providence, RI, (1998)

[GG] Gol'dsheid, I. Ya. and Guivarc'h, Y., Zariski closure and the dimension of the Gaussian law of the product of random matrices. I, Probability Theory and Related Fields, 105, (1996)

[GM] Gol'dsheid, I. Ya. and G.A. Margulis, Lyapunov exponents of a product of random matrices. (Russian) Uspekhi Mat. Nauk 44, (1989), no. 5 (269), 13-60; translation in Russian Math. Surveys 44, (1989),11-71

[GR] Guivarc'h, Y. and Z. Raugi, Propriétés de contraction d'un semi-groupe de matrices inversibles. Coefficients de Liapunoff d'un produit de matrices aléatoires indépendantes, Israel J. Math., 65, (1989), 165-196

[HJ] Horn, R.A. and Ch.R. Johnson, Matrix analysis, Corrected reprint of the 1985 original, Cambridge University Press, Cambridge, (1990)

[M] Margulis, G.A., Discrete subgroups of semisimple Lie groups, Ergebnisse der Mathematik und ihrer Grenzgebiete (3), Springer-Verlag, Berlin, (1991)

[MS] Margulis, G.A. and G.A. Soifer, Maximal subgroups of infinite index in finitely generated linear groups, Journal of Algebra, 69, (1981), 1-23

[N] Noskov, G., Hilbert geometry and dynamics of non-expanding maps, Preprint 2005.

[Pl] Platonov, V.P., Proximal Elements in p-adic Groups SFB343-Bielefeld Preprint 94-099

[P] Prasad, G., R-regular elements in Zariski-dense subgroups, Quart. J. Math. Oxford Ser. (2), 45, (1994), 541-545

[PRa] Prasad, G. and M.S. Raghunathan, Cartan subgroups and lattices in semi-simple groups, Ann. of Math. (2), Annals of Mathematics. Second Series, 96, (1972), 296317 
[PR1] Prasad, G. and A.S. Rapinchuk, Zariski-dense subgroups and transcendental number theory, Math. Res. Lett., 12, (2005), 239-249

[PR2] Prasad, G. and A.S. Rapinchuk, Existence of irreducible $\mathbb{R}$-regular elements in Zariskidense subgroups, Math. Res. Lett., 10, (2003), 21-32

[S] Soifer, G.A., Free subgroups of Linear Groups, a survey, in this volume

[T] Tits, J., Free subgroups in Linear Groups, J. of Algebra 20 (1972) 250-270.

Herbert Abels

Universität Bielefeld

Fakultät für Mathematik

Postfach 100131

33501 Bielefeld

Germany

E-mail: abels@math.uni-bielefeld.de 